\title{
RED SOCIAL ONLINE PARA LOS ABOGADOS
}

\author{
Guadalupe Aleida VALENZUELA MIRANDA ${ }^{9}$
} Darwin MELLADO DURAN ${ }^{10}$

\begin{abstract}
SUMARIO: I. Resumen. II. Introducción. III. Objetivos Generales. IV. Objetivos Específicos. V. Antecedentes VI. Justificación. VII. Relevancia. VIII. Metas. IX. Retos. X. Diseño. XI. Viabilidad. XII. Formación de Recursos Humanos. XIII. Conclusiones. XIV. Bibliografía. XV. Anexos.
\end{abstract}

\section{RESUMEN}

El trabajo aborda temas y problemáticas que actualmente son materia de discusión dentro de la sociedad actual. Se presenta una propuesta de trabajo en Redes Online para las instituciones, empresas o negocios que requieran profesionistas del derecho egresados de la universidad de Sonora a través de su saber- hacer. A lo largo de su historia la Universidad de Sonora ha venido desarrollado mecanismos y estrategias que le permitan establecer vínculos estrechos y de retroalimentación con la sociedad, la comunidad universitaria y el sector productivo, buscando de esta manera la plena divulgación e intercambio de ideas sobre los avances y proyectos que surgen y se generan dentro de la propia Institución, con el fin de enfrentar los retos y cambios constantes que demanda la sociedad.

\footnotetext{
${ }^{9}$ Doctora en Educación por la UNED. Investigador de Tiempo Completo, Categoría Titular "A". Pertenece al Grupo de Investigación en Estudios en Ciencias Sociales CIECS. Departamento de Derecho Universidad de Sonora México. http://www.ciiacs.uson.mx Email: avalenzuela@sociales.uson.mx.

${ }^{10}$ Estudiante de la Universidad de Sonora.snofia@hotmail.com
} 
El objetivo principal de este proyecto consiste en: Difundir un modelo de red social online universitaria, que permita divulgar e intercambiar información relacionada con los proyectos, actividades e investigaciones realizadas por los profesionales del Derecho de la Universidad de Sonora. Los Hallazgos muestran que en los estudiantes universitarios, las redes sociales han creado espacios particulares representando un punto de encuentro entre personas en el que los fines académicos aunque incipientes, constituyen significativamente un sistema abierto que se va construyendo y redefiniendo progresivamente conforme a la incorporación de nuevos miembros y la creación de grupos de trabajo con intereses en común que permite la inclusión de los profesionistas aprovechando los nuevos esquemas de comunicación.

\section{INTRODUCCIÓN}

Algunas personas pueden pensar que no necesitan la tecnología para que les enseñe como "aprenden a aprender", es decir como las personas se interrelacionan y responden a sus propios aprendizajes. En la actualidad hay que buscar métodos que nos permitan un proceso exitoso del aprendizaje para adquirir nuevos conocimientos, técnicas y habilidades que conduzcan al descubrimiento de nuevas formas de trabajo y auto dirigir la propia práctica profesional.

Esta sugerencia es de gran utilidad para aquellas personas dispuestas a cambiar y encontrar un buen empleo, la información acerca de la Red Social online, es un tema muy discutido por los expertos, tenga la seguridad de que aprenderá una o dos cosas... o más. Además de enseñarle cómo aprender y ponerle sazón a su forma de trabajar, también le mostrará los aspectos básicos para trabajar a través de una Red Inteligente.

Las últimas investigaciones han dado como resultado un nuevo enfoque sobre como los seres humanos aprendemos; no existe una sola forma de aprender, cada persona tiene una forma particular de establecer relaciones con el mundo y por lo tanto para aprender a trabajar.

En la primera parte del trabajo se presentan algunos puntos clave, objetivos, justificación y relevancia. En la segunda, se revisan algunos fundamentos teóricos que 
discuten el tópico. En la tercera, se explica la propuesta, metodología y viabilidad, en la última parte, se proporciona un cuestionario y un glosario de términos. Estos elementos permitirán a los profesores, tutores, orientadores o investigadores e institución, fortalecer los vínculos con el sector productivo.

\section{OBJETIVOS GENERALES}

- Promover e impulsar en los estudiantes, profesores e investigadores su participación académica en proyectos de vinculación a través de las "REDES SOCIALES ONLINE" para las empresas o negocios que requieran profesionistas del Derecho de la universidad de Sonora.

- Fomentar en los estudiantes del derecho los nuevos retos a los que se enfrentaran en su desempeño profesional, a partir de los actuales escenarios sociales, la transformación de las instituciones del derecho y su adaptación a los desafíos que plantea la sociedad moderna del siglo XXI, conocida también como sociedad Red.

\section{OBJETIVOS ESPECÍFICOS}

- Establecer un espacio de comunicación para la comunidad en general de los resultados de la formación académica, investigaciones jurídicas realizadas por los expertos del Derecho egresados de la Universidad de Sonora.

- Difundir la práctica profesional del abogado a través del soporte en online

- Socializar y convivir con los profesionales de otras disciplinas e instituciones, a través de las RED Social en línea.

- Fortalecer vínculos de trabajo entre estudiantes, profesores e investigadores de la Universidad de sonora con el sector productivo.

- Mejorar la comunicación entre la comunidad estudiantil y laboral

- Globalizar, a nivel institución, la generación y divulgación de conocimiento

- Proporcionar documentación y gestión administrativa. 
- Crear una Red Social Inteligente Online, con el respaldo institucional, cuya producción académica y científica estará al servicio de la sociedad y sus instituciones

- Proporcionar una herramienta de publicación en línea en el que se den a conocer su desempeño profesional, la competencia laboral, los avances en materia del derecho y sus tendencias más actuales.

\section{ANTECEDENTES}

Los cambios tecnológicos de los últimos años han comportado cambios drásticos en las características de la comunicación en línea y, como consecuencia, empieza la exigencia en las instituciones de educación superior de implementar nuevas estrategias que ajusten su forma de crear, compartir y preservar conocimientos.

Hoy en día, la Redes Sociales su potencial creativo y activo han penetrado en las instituciones de Educación Superior, por lo que el profesorado tiene el reto de traspasar ese tipo de barreras que provocan los cambios y producen un entorno comunicativo a través de aplicaciones sociales disponibles en la red. La verdadera transformación se encuentra en la dinámica educativa, en el proceso educativo que se desarrollan en el aula y, hoy cada vez más, fuera de ella, (Duart, Josep M, 2009). Este nuevo modelo fomenta la colaboración y participación de los estudiantes con estrategias de aprendizaje participativo con un método innovador, (Herrington y Herrington, 2005).

La incorporación de las nuevas tecnologías a la Educación Superior ha sido ampliamente argumentada, por UNESCO (2009) donde se plantea que la aplicación de las TIC a la enseñanza y el aprendizaje encierran un gran potencial para incrementar el acceso, la calidad y el aprovechamiento de los estudiantes de Educación Superior. Sin embargo aun no se tiene una visión clara sobre la aportación que las redes sociales, el cual forman parte de esta tecnología potencial que UNESCO ha planteado, y se argumenta el motivo de esta falta de visión, es debido a la complejidad al momento 
regular como un medio que aporte a la educación y a los procesos de enseñanza aprendizaje.

\section{JUSTIFICACIÓN}

En la actualidad, la sociedad ha cambiado y su forma de comunicare también, las relaciones entre amigos, colegas y familiares se han ido modificando a tal grado que la interacción entre las personas se origina cada vez más en las redes sociales online que en la propia interacción personal (face-to-face). Convirtiéndose de esta manera en un fenómeno social que revoluciona la forma de comunicarse y de interactuar.

Debido a estas ventajas creemos que el desarrollo de una Red social online dentro de la Universidad de Sonora, especialmente para los profesionales del Derecho, permitirá difundir y generar conocimientos que facilitan el crecimiento de la institución y de su propia profesión. Por lo tanto, la innovación radica en proponer el uso de una red social online como herramienta de vinculación empresarial para los abogados de la universidad de Sonora, que permita la divulgación y vinculación de las actividades concernientes de la institución con la sociedad y el sector productivo. Además de esto, nuestra propuesta consiste en la aplicación de un modelo de seguridad basado en agentes inteligentes el cual sería un aporte a la innovación de redes sociales online. Este modelo permitirá el desarrollo de redes sociales basadas en agentes inteligentes que garanticen una mejor generación y diseminación de conocimiento. En la actualidad las redes sociales se abren como nuevos canales de información y de aprendizaje que se hacen imprescindibles para el desarrollo profesional, sin embargo, muchos docentes, aun no ven estas tecnologías como un instrumento de mejora continua.

Indiscutiblemente es reconocido por expertos que las redes sociales son aquellos medios que facilitan el aprendizaje, la comunicación, el intercambio de conocimiento entre cualquier persona o grupo que participan en ellas, generando la posibilidad y la 
alternativa para abrir nuevos horizontes en busca del desarrollo de estrategias de enseñanza, enfocadas a las nuevas exigencias del trabajo.

En suma, las redes sociales cumplen ya un papel fundamental en el desarrollo humano, desde nuestro punto de vista, de favorecer una actitud positiva en los actores del proceso educativo, contemplando los medios emergentes de comunicación hacia su incorporación en los procesos laborales, educativos e institucionales. Característica que se espera contribuya a una profunda actualización de los métodos de enseñanza, potenciando la participación activa de los estudiantes de la disciplina o carrera y una aproximación entre la educación formal e informal, de modo que desde la escuela se afronten los retos de enseñar a ser críticos ante la información que nos llega por medios cada vez más sofisticados y potenciar la capacidad de análisis y reflexión que dotará de sentido a los datos recibidos de forma dispersa para alcanzar un conocimiento integrado, que posteriormente se convertirá en el nuevo capital humano del abogado

\section{RELEVANCIA}

El trabajo permitirá diagnosticar sobre la utilización que dan los estudiantes de la Universidad de Sonora a las redes sociales, particularmente en actividades concernientes a la búsqueda de empleo con la sociedad y la industria, cuyo resultado permitirá conocer la situación en que se encuentra el desarrollo e incorporación de las nuevas tecnologías en la educación superior, a fin de lograr la participación de las personas involucradas en el tópico, en función de las tendencias más actuales en materia de derecho, en cuanto a su uso en el sector productivo. Asimismo es posible identificar aspectos relevantes relacionados a la formación necesaria en el profesorado y la generación de recomendaciones y propuestas de innovación que contribuyan a la generación de procesos de aprendizaje que estimulen al estudiante, para su futuro 
desempeño laboral ó profesional. Es decir, gracias a la participación en Redes Sociales en Online, los estudiantes pueden desarrollar su capital humano, y con ello mejorar su economía.

\section{METAS}

1.- Se espera generar un medio de difusión y aplicación del conocimiento para atender la problemática de empleo en la sociedad

2.-Desarrollar el capital humano a través de estas Redes Sociales

3.-Newsletters como Redes sociales o Comunidades Virtuales de participación ciudadana

4.-Realizar cursos de capacitación al personal que participe en la Red Social

Online para explotar las herramientas web 2.0 y 3.0

5.-Invitar a los migrantes Digitales a socializar en la RED, como una nueva forma de encontrar empleo.

6.- Adquirir los insumos necesarios para la implementación del proyecto de manera institucional.

\section{RETOS ACTUALES}

- Ser un migrante digital para entender a los nativos digitales que nacieron con el siglo 
- Potenciar el conocimiento intelectual como una forma de desplegar el capital Humano

- Necesidades de capacitarse en el uso y aplicaciones.

- Diferencias entre la visión y la Acción en la formación de Recursos Humanos

- No es posible transmitir conocimiento de forma unidireccional.

- Admitir que la Red es un gran apoyo para la construcción colaborativa de conocimiento.

- Ser permeable a los cambios laborales que se producen en el entorno actual y de los usos laborales de la red.

- Las redes sociales deben penetrar en la institución, para convertirse en la base de una política institucional.

- Una nueva cultura de usuarios activos capaces de crear, modificar, buscar, comunicar y compartir información y conocimiento.

- Un cambio de paradigma, con nuevas estrategias y metodologías didácticas innovadoras para conseguir empleo.

- Debe de ser una realidad en la sociedad para las empresas que les interese contratar abogados o profesionales del Derecho.

- Disminuir la brecha digital entre profesionista y el empleador

- El uso activo y social de la Red no puede ser ignorado en las planificaciones docentes, principalmente cuando se trata de vincular al sector productivo, llámese empresas, negocios, corporativos, transnacionales y demás actores sociales.

\section{Diseño cuantitativo: Investigación exploratoria.}

Los estudios exploratorios se realizan cuando el problema de investigación es poco estudiado del cual se tienen muchas dudas, o bien no se han abordado antes. Indagan 
desde una perspectiva innovadora, ayudan a identificar conceptos y preparan el terreno para nuevos estudios.

\section{Fases del Diseño:}

Fase 1. Planeación, Diseño de las Estrategias de trabajo y calendarización de los tiempos

Fase 2. Recolección de información y observaciones

Fase 3. Aplicaciones web 2.0 (Red Social inteligente Online, SNOFIA)

Fase 4. Análisis y Ajuste del diseño al contexto

Fase 5. Captura

Fase 6. Análisis Estadístico de los datos (software estadístico PASW )

Fase 7. Elaboración de conclusiones y presentación de resultados

Contexto: Licenciatura en Derecho, Universidad de Sonora, México.

\section{VIABILIDAD}

El estudio no presenta ningún problema de viabilidad, pues diversas empresas están muy interesadas en establecer su presencia en las Redes Sociales. De hecho, se plantea que a cambio de que permitan conducir la investigación en nuestro contexto, se les entregue un reporte que describa al mercado laboral existente y se analicen las propuestas de trabajo o empleo, con el sector productivo.

\section{FORMACIÓN DE RECURSOS HUMANOS}


Se espera con el Proyecto lograr asesorar al menos a cinco estudiantes de la licenciatura en Derecho y otros más de Maestría y dos de doctorado, como colaboradores voluntarios del proyecto.

\section{CONCLUSIONES}

Los profesionales del derecho deben tener más presentes las posibilidades innovadoras que nos ofrecen las redes sociales para adecuar en su práctica profesional, los retos que enfrenta su nuevo perfil con el fin de lograr las promesas y expectativas que la cultura tecnológica trata de propagar en el mundo laboral. Por lo tanto, el gran reto es lograr que los profesionistas utilicen las redes sociales con fines laborales, estableciendo vínculos de trabajo entre estudiantes, profesores e investigadores de la Universidad de Sonora con el sector productivo.

Finalmente, este tipo de propuestas tecnológicas e innovadoras se enfocan en la producción de nuevo conocimiento útil en la práctica que se obtiene mediante el cambio y/o búsqueda de soluciones a situaciones emergentes que le ocurren a un grupo de personas interesados en discutir nuevas formas de representación laboral. Los resultados de esta experiencia deben ser provechosos tanto para el investigador como para los participantes y la misma Institución.

Quiero terminar este trabajo citando una frase muy conocida y repetida por algunos teóricos en sus aportaciones, cuando reconocen que "La información es la sangre que da vida al conocimiento, y las Redes Sociales son sus venas. 


\section{BIBLIOGRAFÍA}

1.-BRUNNER, JOSÉ Joaquín. Globalización y el futuro de la educación. Tendencias, desafíos y estrategias. Seminario sobre prospectiva de la educación en la región de América Latina y el Caribe. UNESCO. Santiago de Chile. (2000).

2. GARIJO, F. Tecnología de Agentes: Experiencias y perspectivas para el desarrollo de nuevos servicios y aplicaciones .Boletín No. 24, PP.: 1-9. (2002).

3.- DUART, JOSEP M. Internet, redes sociales y educación. RU\&SC. Revista de Universidad y Sociedad del Conocimiento, Vol. 6, Núm. 1, marzo, 2. Universitat Oberta de Catalunya Catalunya, España,(2009).

4.- GALLEGO DOMINGO José. y ALONSO. CATALINA María. Cuestionario de Redes Sociales para el Aprendizaje (CRESAP). 6.-Universidad de Education a Distancia (UNED). Madrid, España, (2010).

5.-HARGITTAI, E., Whose space? Differences among users and non-users of social network sites. Journal of Computer-Mediated Communication, Vol. 13 (1). 2007.

6.-HERRINGTON, A. y HERRINGTON, J. Authentic learning environments in higher education. Hershey, PA: Information Science Publishing. (Eds). 2005.

7.- VAN ALSENOY, B., Ballet J., Kuczerawy, A., Dumortier, J., Social networks and web 2.0: are users also bound by data protection regulations?, en Identity in the information society, Vol. 2(1), Pp. 65-79. 2009.

8.-. WOOLDRIDGE, M., Jennings, N.R" Intelligent Agents: Theory and Practice." Knowledge Engineering Review, Vol. 10, No. 2, pp: 115-152. 1995.

9.-SIMÕES, LUIS; BORGES GOUVEIA, Luis "Web 2.0 and Higher Education

Pedagogical Implications". Proceedings of the 4th International Barcelona Conference on Higher Education, Vol. 12. Knowledge technologies for social transformation. Barcelona: GUNI. 2008

10.-UNESCO "Conferencia Mundial sobre la Educación Superior. La nueva dinámica de la educación superior y la investigación para el cambio social y el desarrollo" Sede UNESCO. Documento. Paris, 2009.

\section{ANEXOS}

1.- Instrumento. CRESAP. Nuevas Tecnologías y Redes Sociales. Rediseñado por Adrià Velia González, Guadalupe Aleida Valenzuela Miranda, Adolfo Castillo, Edgar González Bello, y Domingo Gallego. (2010) Ver en:

https://spreadsheets.google.com/viewform?formkey=dERaTU9jUUNySnRZNEpob1RtU HUWWIE6MQ

Fecha e consulta: 07/febrero/2011. 


\section{GLOSARIO DE TÉRMINOS}

TÍTULO: Enunciado de una o dos líneas que define la temática a realizar de manera concisa. Debe ser claro, reflejar el tema en particular, el área de estudio y los alcances de la investigación.

RESUMEN: Definición que expresa de manera clara y precisa todo sobre el proyecto a realizar. Responde al ¿qué?, ¿cómo? y ¿para qué? La definición del proyecto debe ser clara, precisa y concisa.

ANTECEDENTES: Se refiere a la información e investigaciones previas relacionadas con el proyecto. Deben presentar las generalidades sobre el tema, que apoyen y fundamenten la investigación, que sean actualizados y congruentes con el proyecto.

JUSTIFICACIÓN: Expresa las razones para demostrar que la investigación se debe de llevar a cabo. Debe ser real, clara y precisa.

OBJETIVOS: Expresa la declaración de intención que especifica con claridad el "que” y el "para que” se requiere realizar la investigación. Deben ser claros, precisos y congruentes con las metas y con el proyecto mismo.

METAS: Es la expresión precisa de los fines o resultados que se pretenden alcanzar con la realización del proyecto de investigación, fijando y determinando tiempos y recursos. Debe de redactarse de manera tal que pueda usarse como medición de calidad durante la evaluación. Deben ser cuantificables, realistas, claras, precisas y congruentes con el proyecto. 
METODOLOGÍA: Procedimiento o pasos que se han de seguir para llevar a buen término la investigación. Debe ser clara, detallada y consecuente con la realización del proyecto y para el cumplimiento de los objetivos y metas.

BIBLIOGRAFÍA: Apartado que presenta las citas bibliográficas de donde se obtuvo la información para la construcción de los distintos apartados de la propuesta. Debe estar actualizada y adecuada al proyecto.

ANEXOS: Información que complemente la investigación y apoye la evaluación de la propuesta. 